\title{
COUPLING IMPEDANCE OF A PERIODIC ARRAY OF DIAPHRAGMS
}

\author{
G. V. Stupakov, Stanford Linear Accelerator Center, Stanford University, Stanford, CA 94309 USA
}

\begin{abstract}
A method is presented for calculating the high-frequency longitudinal and transverse coupling impedances in a periodic array of diaphragms in a circular perfectly conducting pipe. The method is based on Weinstein's theory of diffraction of a plane electromagnetic wave on a stack of halfplanes. Using Weinstein's solution, it is shown that the problem of finding the beam field in the pipe reduces to an effective boundary condition at the radius of the diaphragms which couples the longitudinal electric field with the azimuthal magnetic one. Solving Maxwell's equations with this boundary condition leads to simple formulae for $Z_{\text {long }}$ and $Z_{t r}$. A good agreement with a numerical solution of the problem found by other authors is demonstrated.
\end{abstract}

\section{INTRODUCTION}

Studies of the impedance at the frequencies much higher than the cutoff frequency have a long history with many theoretical and numerical results obtained for different types of accelerator structures (see e.g. a special issue of Particle Accelerators journal devoted exclusively to this subject [1]). One of the major problems addressed by several authors is the highfrequency impedance of multiple cavities or a periodic system of diaphragms [2-5]. There is a general consensus that, for large $\omega$, the longitudinal impedance in this system scales asymptotically as $\omega^{-3 / 2}$. Specifically, for a periodic array of thin diaphragms, in the limit $\omega \rightarrow \infty$, the real part of $Z_{\text {long }}$ per one cell can be approximated by the following function:

$$
\operatorname{Re} Z_{\text {long }}(\omega) / Z_{0} \approx f(g / a)^{3}(k g)^{-3 / 2},
$$

where $k=\omega / c, Z_{0}=4 \pi / c=377 \Omega, g$ is the distance between the diaphragm openings, $a$ is the radius of the diaphragms, and $f$ is a numerical factor. However, various authors find different values for $f$ which deviate almost by the order of magnitude: $f=\pi^{-1 / 2}$ in Ref. [3], $f=8 \pi^{-1 / 2}$ in Ref. [4], and according to the Sessler - Weinstein model [2], $f=0.67 \pi^{-1 / 2}$.

Apart from differing numerical values for $f$, Eq. (1) itself gives a rather poor approximation in the region $10<k g<20$ typical for practical applications in accelerator physics. The reason for Eq. (1) to be relatively inaccurate is that the actual parameter in asymptotic expansion (1) is $(\mathrm{kg})^{1 / 2}$ (or even $(\mathrm{kg} / \pi)^{1 / 2}$ ) rather than $\mathrm{kg}$. This makes it necessary to seek better asymptotes than the leading term represented by Eq. (1). Refs. [2-3] indeed provide a more accurate expressions that reduce to Eq. (1) in the limit $(k g)^{1 / 2} \gg 1$.

In this paper an attempt is made to revise the approach to the calculation of the impedance of the periodic system of diaphragms using a more adequate physical description of the beam interaction with the diaphragms. On a qualitative level, the physics involved has been outlined in Ref. [6]. Its two basic elements are: a small angle diffraction of the beam field at the edges of the diaphragms, and depletion of the amplitude of the field in the region close to the edges due to repeated trapping of the field energy into the space between the diaphragms. We will show that this qualitative argument can be cast into a quantitative consideration using a rigorous solution to the diffraction of a plane electromagnetic wave on an infinite stack of conducting halfplanes.

\section{BASIC ASSUMPTIONS}

Consider a relativistic beam with a factor of $\gamma$ much larger than unity, $\gamma \gg 1$, propagating along the axis of a circular pipe with infinitely thin periodic diaphragms. The azimuthal magnetic field of such a beam is almost equal to its radial electric field, and both propagate with the speed $\approx c$. In that respect, excluding the vicinity of the axis of the pipe occupied by the beam, the electromagnetic field can be considered as a free electromagnetic wave propagating in the pipe. Accepting this point of view, we intend to apply to the beam field the results derived from the diffraction of the wave on the edges of the diaphragms.

The analysis of the diffraction is greatly simplified by the fact that we are only interested in the high frequency band. From Fresnel theory of diffraction, it is known that the area involved in the diffraction extends from the edges by distance $\sim \sqrt{g / k}$, and occupies an annulus from $r \approx a-d \sqrt{g / k}$ to $r \approx a+d \sqrt{g / k}$, where $d$ has a value of the order of unity. As soon as $\sqrt{g / k}$ is much smaller than the radius $a$, we can neglect the cylindrical geometry of the problem and consider the diffraction in plane geometry. We will also assume that $\sqrt{g / k} \ll b-a$, where $b$ is the pipe radius; in this case the pipe wall does not interfere with the diffraction process, and we can further simplify the problem eliminating the pipe walls and allowing the field to freely propagate in the radial direction to infinity [5].

As a result of these approximations we essentially reduce the problem to the diffraction of a plane electromagnetic wave on an infinite periodic array of halfplanes the solution for which can be found in Ref. [7].

\section{WEINSTEIN'S THEORY}

This section briefly summarizes Weinstein's results for the diffraction of a plane wave for an arbitrary incidence angle $\varphi_{0},\left(\varphi_{0}\right.$ is measured from the vertical axis $y$ so that the grazing incidence corresponds to $\varphi_{0}=\pi / 2$ ). In our case, the beam field propagates horizontally which corresponds to the limit $\cos \varphi_{0} \rightarrow 0$ in the diffraction solution.

Let the position of $m$ th halfplane be given by $z=m g, y \leq 0$. Consider a plane wave propagating in the halfspace $y>0$ at an angle $\varphi_{0}$ with the vertical axis $\left(0 \leq \varphi_{0} \leq \pi / 2\right)$ and polarized so that the only component of the magnetic field is directed along the $x$-axis,

$$
H_{x}=A \exp \left[i k\left(z \sin \varphi_{0}-y \cos \varphi_{0}\right)\right] .
$$

Here and below we assume the time dependence $\propto \exp (-i \omega t)$. 
The solution to the diffraction problem for the incident wave (2) [7, Chapter 7] represents the field at $y<0$ as a sum of eigenmodes propagating between the plates:

$$
H_{x}=A\left(T_{0} e^{-i k y}+\sum_{n=1}^{\infty} T_{n} \cos \frac{\pi n z}{g} e^{-i \kappa_{n} y}\right)
$$

where $\kappa_{n}=\sqrt{k^{2}-(\pi n / g)^{2}}, \operatorname{Im} \kappa_{n} \geq 0$. Eq. (3) is valid for $0<z<g$; the field between $m$ th and $(m+1)$ th plates has an additional factor $\exp \left(i \mathrm{kmg} \sin \varphi_{0}\right)$ on the right hand side. Complex values of $\kappa_{n}$ imply that the corresponding eigenmode is an evanescent one; it exponentially decays when $y \rightarrow-\infty$.

The field in the upper halfspace, $y>0$, is given by

$$
\begin{array}{r}
H_{x}=A e^{i k\left(z \sin \varphi_{0}-y \cos \varphi_{0}\right)} \\
+A \sum_{n=-\infty}^{\infty} R_{n} e^{i k\left(z \sin \varphi_{n}+y \cos \varphi_{n}\right)},
\end{array}
$$

where $\cos \varphi_{n}=\left[1-\left(n+q \sin \varphi_{0}\right)^{2} / q^{2}\right]^{1 / 2}, q=k g / 2 \pi$; it is assumed that $\operatorname{Im}\left(\cos \varphi_{n}\right) \geq 0$. The first term on the right side of Eq. (4) is the incident wave, and the sum represents the diffracted waves generated by the periodic structure. One of these waves having $n=0$ is a mirror reflected image of the incident field; it has the amplitude $A R_{0}$.

The expressions for $T_{n}$ and $R_{n}$ can be found in Ref. [7]. For our purposes, we will only need $R_{0}$ as a function of $q$ and $\varphi_{0}$,

$$
\begin{array}{r}
R_{0}\left(q, \cos \varphi_{0}\right)=-\frac{1-\cos \varphi_{0}}{1+\cos \varphi_{0}} e^{4 i q \cos \left(\varphi_{0}\right) \ln 2} \times \\
\prod_{n=1}^{\infty} \frac{1+\frac{\cos \varphi_{0}}{\cos \varphi_{n}}}{1-\frac{\cos \varphi_{0}}{\cos \varphi_{n}}} \frac{1+\frac{\cos \varphi_{0}}{\cos \varphi_{-n}}}{1-\frac{\cos \varphi_{0}}{\cos \varphi_{-n}}} \frac{1-\frac{2 \pi q \cos \varphi_{0}}{\kappa_{n} g}}{1+\frac{2 \pi q \cos \varphi_{0}}{\kappa_{n} g}} .
\end{array}
$$

\section{BOUNDARY CONDITION}

To consider the case of horizontal propagation of the wave we need, first, to find the limit $\cos \varphi_{0} \rightarrow 0$ in Eq. (5). Using analysis of Ref. [7], after straightforward though cumbersome algebra, one can show that in this limit, for $k g \gg 1$,

$$
R_{0}=-1+2 S(k g) \cos \varphi_{0},
$$

where the complex function $S(q)$ is given by

$$
S(x)=\frac{1}{2}\left[1+\frac{(1-i)}{\pi} \sqrt{\frac{x}{2}}((\sqrt{2}-1) F(2 x)+\alpha)\right],
$$

where

$$
F(x)=\int_{-\infty}^{\infty} d t\left(\exp \left(\frac{t^{2}}{2}-i x\right)-1\right)^{-1}
$$

and

$$
\alpha=-2 \int_{-\infty}^{\infty} \frac{1-e^{t^{2} / 2}+t^{2} / 2}{t^{2}\left(e^{t^{2} / 2}-1\right)} d t=3.658
$$

The function $F(x)$ is a periodic function of its argument with the period equal to $2 \pi$. It has singularities $\propto|x-2 m \pi|^{-1 / 2}$ at the points $x=2 m \pi$, where $m$ is an integer.
Turning now to the physical interpretation of the solution, note that in the limit $\cos \varphi_{0} \rightarrow 0$, both the incident wave given by Eq. (2) and the mirror reflected wave $A R_{0} \exp \left(i k\left(z \sin \varphi_{0}+y \cos \varphi_{0}\right)\right)$ in Eq. (4) propagate parallel to the horizontal axis. This observation prompts us to believe that their sum has to be identified with the electromagnetic field of the beam at the edge of the diaphragms. Using Eq. (6) we find for the magnetic component of this field:

$$
\begin{array}{r}
H_{x}=A e^{i k z \sin \varphi_{0}}\left(e^{-i k y \cos \varphi_{0}}+R_{0} e^{i k y \cos \varphi_{0}}\right) \approx \\
A e^{i k z}\left(-2 i \sin \left(k y \cos \varphi_{0}\right)+2 S(k g) \cos \varphi_{0} e^{i k y \cos \varphi_{0}}\right) \approx \\
2 A \cos \varphi_{0} e^{i k z}(S(k g)-i k y) .
\end{array}
$$

In order to obtain a nonzero result when $\cos \varphi_{0} \rightarrow 0$, we have to assume that $A$ goes to infinity so that $2 A \cos \varphi_{0} \rightarrow E$ and

$$
H_{x}=E e^{i k z}(S(k g)-i k y),
$$

where $E$ is a constant. We see that our solution requires the magnetic field to be a linear function of $y$; in other words, for $\varphi_{0}=\pi / 2$ the diffraction process imposes a certain constraint on the behavior of the electromagnetic field near the edges of the diaphragms. This constraint can be expressed as a boundary condition at $y=0$ if one notes that Maxwell's equation $\partial H_{x} / \partial y=i k E_{z}$ combined with Eq. (11) allows to one express $E$ in terms of the electric field: $E_{z}=-E \exp (i k z)$. Substituting this relation in Eq. (11) yields

$$
E_{z}=-\left.\frac{1}{S(k g)} H_{x}\right|_{y=0} .
$$

Eq. (12) represents our main result. It relates the longitudinal component of the electric field to the transverse component of the magnetic field at the diaphragms.

Note a close resemblance of Eq. (12) to the boundary condition at a conducting wall in the case of high conductivity $\sigma$, $E_{z}=\left.(i-1) \sqrt{\omega / 8 \pi \sigma} H_{x}\right|_{y=0}$, [8]. This allows us to assign the diaphragms an effective (complex) conductivity $\sigma_{\text {eff }}$, such that

$$
\sqrt{\frac{2 \pi \sigma_{\mathrm{eff}}}{\omega}}=\frac{(i-1)}{2} S(\mathrm{~kg}) .
$$

Using Eq. (13), for a given solution of an electromagnetic problem in a smooth pipe with finite conductivity $\sigma(\omega)$, one can find the solution of the corresponding problem in the pipe with periodic diaphragms by substitution $\sigma \rightarrow \sigma_{\text {eff }}$.

\section{IMPEDANCE}

Having found the boundary condition (12) we can now return to the cylindrical geometry of the pipe with the beam and solve for the beam field in the region $0<r<a$. In polar coordinate system, the $x$-component of the magnetic field $H_{x}$ should be identified with the azimuthal component $H_{\vartheta}$, so that Eq. (12) takes the form,

$$
E_{z}=-\left.\frac{1}{S(k g)} H_{\vartheta}\right|_{r=a}
$$

With this boundary condition, a standard derivation (see, e.g., [9]) of the longitudinal and transverse impedances, $Z_{\text {long }}$ and $Z_{t r}$, 


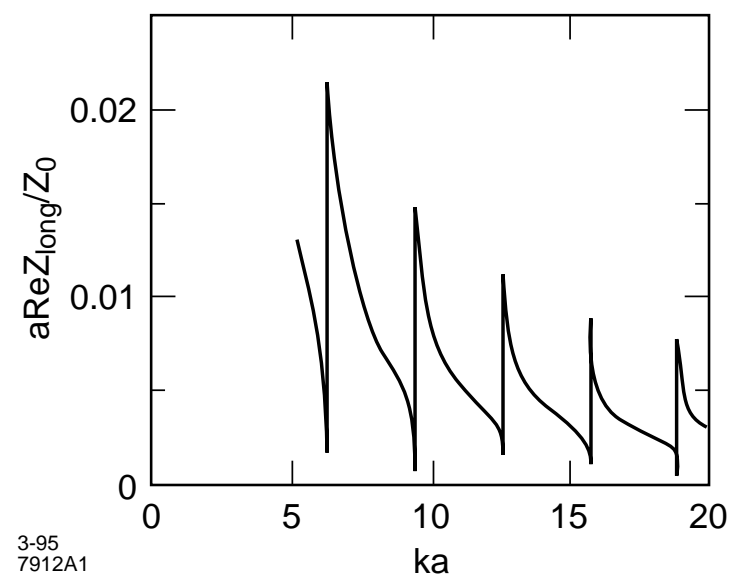

Figure. 1. Real part of the longitudinal impedance.

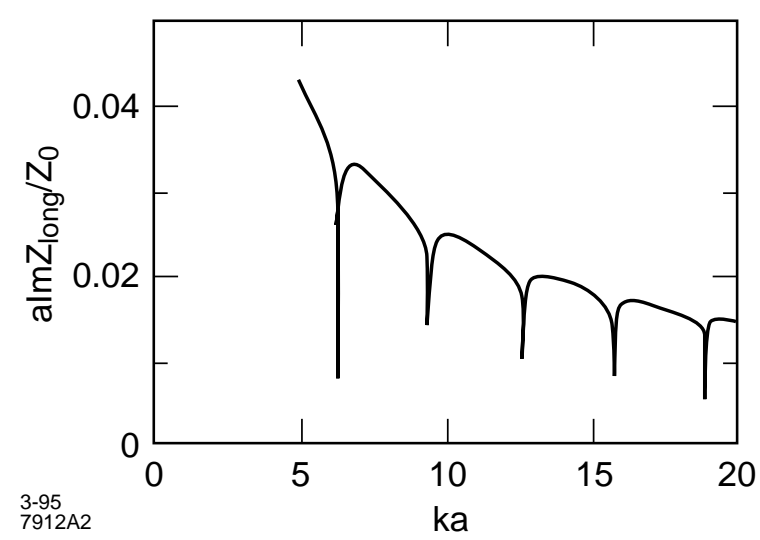

Figure. 2. Imaginary part of the longitudinal impedance.

yields :

$$
\begin{array}{r}
Z_{\text {long }}=\frac{Z_{0}}{2 \pi a} \frac{1}{S(k g)-i k a / 2}, \\
Z_{t r}=\frac{Z_{0}}{\pi k a^{3}} \frac{1}{S(k g)-\frac{1}{2} i k a+i(k a)^{-1}} .
\end{array}
$$

Figures 1 and 2 show the real and imaginary parts of $Z_{\text {long }}$ for the case when $a=g$. In addition to general fall off of $Z_{\text {long }}$ with the frequency, it demonstrates peaks and jumps at $k a=m \pi$, where $m$ is an integer. This behavior can be explained as due to a strong coupling, through diffraction, of the beam field with the modes between the diaphragms having a small radial wavenumber. These modes have the frequency close to $\pi \mathrm{m} / \mathrm{a}$; they represent standing waves between two adjacent diaphragms.

In the limit of very high frequency, $\omega \rightarrow \infty$, the asymptotic dependence of $Z_{\text {long }}(\omega)$ is given by

$$
\operatorname{Re} Z_{\text {long }} \approx \frac{2 Z_{0}}{\pi k^{2} a^{3}} \operatorname{Re} S(k g), \quad \operatorname{Im} Z_{\text {long }} \approx \frac{Z_{0}}{\pi k a^{2}} .
$$

Note that on the average $\operatorname{Re} Z_{\text {long }}$ scales asymptotically as $\omega^{-3 / 2}$ in agreement with Eq. (1).
Similar to longitudinal impedance, $Z_{t r}$ has sharp peaks at $k a=m \pi$, however, it decays more rapidly than $Z_{\text {long }}(\omega)$. Asymptotically, for $\omega \rightarrow \infty$,

$$
\begin{gathered}
\operatorname{Re} Z_{t r} \approx \frac{4 Z_{0}}{\pi k^{3} a^{5}} \operatorname{Re} S(k g), \operatorname{Im} Z_{t r} \approx \frac{2 Z_{0}}{\pi k^{2} a^{4}} . \\
\text { VI. DISCUSSION }
\end{gathered}
$$

We compared our result with a numerical solution of a similar problem in Ref. [5], where a repeated structure of thin irises has been studied. A close inspection of the plot of $\operatorname{Re} Z_{\text {long }}$ in this reference shows a very good agreement with our Fig. 1, including the positions and the heights of each peak even for $k a$ as small as 5. This agreement indicates that using a plane geometry for solution of the diffraction problem turns out to be a very accurate appoximation even for relatively small values of $k a$.

In the limit of very large frequencies, our result agrees with Eq. (1) with $f=0.26$ which is below both Gluckstern's result $(f=0.56)$ and Sessler-Weinstein model $(f=0.37)$.

\section{ACKNOWLEDGMENT}

I thank Karl Bane, who initiated this work, for many useful discussions, and G. Dome for useful comments.

\section{References}

[1] Part. Accel., 25, (1990)

[2] E. Keil. Nucl. Instr. and Meth., 100, p. 419 (1972).

[3] R.K. Gluckstern. Phys. Rev. D, 39, p. 2780 (1989).

[4] S. A. Heifets and S. A. Kheifets. Rev. Mod. Phys., 63, p. 631-674 (1991).

[5] G. Dome, L. Palumbo, V.G. Vaccaro and L. Verolino. Proceedings of the European Particle Accelerator Conference, Berlin 1992, 2, p. 925 (1992).

[6] R.B. Palmer. Part. Accel., 25, p. 97, (1990).

[7] L.A. Weinstein. The Theory of Diffraction and the Factorization Method (The Golden Press, Boulder, Colorado, 1069)

[8] L.D. Landau and E. M. Lifshits. Electrodynamics of Continuous Media (Pergamon Press, Oxford, 1960).

[9] A. Chao. Physics of Collective Beam Instabilities in High Energy Accelerators (Wiley, New York, 1993). 